Archives

$17 \mid 1996$

Hommage à Bernard Lepetit

\title{
Logiques du sacré et modes d'organisation de l'espace : Le cas du Sud-Ouest algérien
}

Histoire d'une thèse

\section{Abderrahmane Moussaoui}

\section{CpenEdition}

Journals

Édition électronique

URL : http://journals.openedition.org/ccrh/2584

DOI : $10.4000 /$ ccrh.2584

ISSN : 1760-7906

Éditeur

Centre de recherches historiques - EHESS

Édition imprimée

Date de publication : 4 octobre 1996

ISSN : 0990-9141

Référence électronique

Abderrahmane Moussaoui, « Logiques du sacré et modes d'organisation de l'espace : Le cas du SudOuest algérien », Les Cahiers du Centre de Recherches Historiques [En ligne], 17| 1996, mis en ligne le 27 février 2009, consulté le 21 avril 2019. URL : http://journals.openedition.org/ccrh/2584 ; DOI : $10.4000 /$ ccrh.2584

Ce document a été généré automatiquement le 21 avril 2019

Article L.111-1 du Code de la propriété intellectuelle. 


\section{Logiques du sacré et modes d'organisation de l'espace: Le cas du Sud-Ouest algérien}

Histoire d'une thèse

\section{Abderrahmane Moussaoui}

1 Il y a une quinzaine d'années, à l'occasion de la préparation d'un mémoire de licence en sociologie rurale et urbaine, je découvrais le terrain en général et une partie de la région, le Gourara, qui allait faire l'objet de ma thèse. Un des traits particuliers de cette région est son organisation hydraulique. Mise en valeur au moyen d'un système ingénieux, les foggaras, l'eau est privée. Achetée et vendue, c'est elle qui présente un caractère foncier. La terre, elle, suit l'eau, dit l'expression locale. J'avais, alors, construit toute mon approche de l'organisation sociale à travers ce vecteur hydraulique.

2 Il m'a fallu revenir une seconde fois, au milieu des années quatre-vingt, sur le même terrain, en vue de la préparation d'une thèse de troisième cycle, en géographie cette foisci, pour voir que cette eau n'organisait pas seulement le social, elle déterminait également l'aménagement de l'espace.

3 Seulement, la spécialité et la précipitation du jeune chercheur me firent adopter des thèses qui expliquaient tout par les contraintes écosystémiques. Confrontées au réel, à l'aune du monde du travail, ces interprétations - où tantôt le sociologique, tantôt le géographique prenaient le pas sur le reste - se révélèrent inadéquates.

4 Lorsque j'ai travaillé dans un bureau d'études d'urbanisme, j'ai été confronté au décalage existant entre la programmation et la réalité. Les normes officielles découlaient de thèses générales élaborées ailleurs, dans d'autres contextes, et transmises par l'enseignement et les procédés de fabrication. Des grilles d'équipements étaient conçues sur la base de quotas statistiques à partir de moyennes considérées comme universelles. C'était le triomphe de l'urbanisme fonctionnaliste dont les fondements commençaient déjà à être sérieusement remis en cause en Europe. L'échec de la politique des ZUP (zones d'urbanisation prioritaires) et des villes nouvelles, en France, n'empêcha pas les pouvoirs 
publics algériens, pressés par la réalité de la crise du logement et par la poussée démographique, de lancer des programmes standardisés, sans aucune tentative sérieuse d'adaptation au terrain.

5 Hantés par la démographie et confortés par l'apparente et relative facilité d'utilisation du quantitatif, les décideurs fondent tout sur l'outil statistique (problème des catégories statistiques et de leur pertinence, problème de leur justesse et de leur précision). Les statistiques constituaient l'unique critère. Des classes, par exemple, seront programmées sur la base de données statistiques, alors que, sur le terrain, celles qui existaient étaient sous-utilisées ou inutilisées.

Lors des redécoupages administratifs du territoire (1974 et 1982), des communes ont été créées, et des agglomérations érigées en chefs-lieux sur la base de données de topographie et de maillage urbain anciennes. Ce qui entraîne la construction d'un certain nombre d'équipements ainsi que l'ouverture de routes et de voies d'accès. Or, sur le terrain, ces équipements ne se révèlent pas toujours souhaitables et les routes, une fois ouvertes, demeurent rarement ou pas du tout empruntées. La raison en est que l'établissement désigné par des cartes d'état-major dépassées (base de travail des ministères) comme agglomération est en fait déserté depuis longtemps par ses habitants (industrialisation, urbanisation et exode en général). Au même moment, un lieu-dit, que nulle carte ne signale, accroché au flanc d'une montagne, continuait, grâce à la tombe d'un saint, à drainer des foules de visiteurs, à travers des chemins abrupts et des pistes escarpées.

7 C'est ce qui m'amena à m'intéresser davantage à un certain type d'«équipements » comme le cimetière ou la mosquée ; d'autant qu'à leur endroit le silence des normes était (est toujours) complet.

8 Ces constats vont m'amener à essayer de comprendre quelles étaient les véritables logiques déterminant l'organisation, l'aménagement et l'occupation de l'espace. La tombe s'est, tout de suite, révélée comme un des ressorts privilégiés. Pour comprendre l'espace des morts, il a fallu s'intéresser à la mort comme phénomène anthropologique.

9 De plus en plus, la question de la religion et des croyances s'imposait à moi. Ce qui m'amena à l'étude de quelques aspects de la religion, non pas du point de vue doxologique, mais surtout dans son côté vécu. Ce que l'on appelle communément la religion populaire.

Dès lors, mon approche du terrain changea. Désormais, elle se fit à travers le prisme de cet imaginaire enfoui et sédimenté, ne se révélant plus qu'à l'occasion de ce que G. Le Bras appelait « les saisons de la vie». Sous peine de forcer une interprétation d'un donné immatériel ancien et de surcharger le mythe par des conjectures pseudo-scientifiques, il me fallait retrouver le terrain qui porterait encore les traces de logiques de ce type. Il fallait retrouver non pas une épure, mais une sorte de terroir témoin encore travaillé par de telles conceptions.

11 La rapidité des transformations et les structures héritées d'un autre système d'habitat excluaient d'emblée les villes du nord du pays. Leurs noyaux antiques ont été soit détruits, soit totalement transformés et assujettis aux logiques nouvelles imposées par plus d'un siècle de présence coloniale. L'indépendance accélérerait la déstructuration. À l'image de celle d'Alger, les casbahs des médinas se retrouvèrent vite dans un état de dégradation avancé ; leur population a souvent changé et, là aussi, les repères sont devenus flous et le sens se perd chaque jour. 
12 C'est donc presque naturellement que je me suis tourné vers un terrain dont j'avais une première connaissance et qui, malgré une dizaine d'années de distance, est demeuré relativement intact. Il s'agit de Timimoun et de sa région, le Gourara. La ville de Kenadsa, mon second terrain, je la connaissais autrement, étant natif d'une ville distante d'une trentaine de kilomètres. C'est un avantage et un inconvénient à la fois. Les préjugés sont plus forts et l'illusion de connaissance peut empêcher la nécessaire distanciation.

13 S'est posé alors le problème de la méthode d'approche. Mon expérience du terrain me poussa tout de suite à me méfier des approches académiques classiques et des problématiques globalisantes. Ici, le questionnaire écrit et oral, l'interview, sont à manier avec beaucoup de délicatesse.

14 L'approche ethnographique de l'habitat, qui consiste à aller chercher directement dans les formes de l'habitat ce que nous supposons être une origine, est une autre erreur que j'ai soigneusement essayé d'éviter. J'ai voulu une approche qui intègre l'avis des habitants eux-mêmes, tous les habitants, et pas seulement ceux dont la parole est autorisée (Shurfâ et $m r a ̂ b t \hat{n})$.

15 J'ai privilégié un indicateur qui m'a semblé pertinent. Il s'agit de la fête du mawlid. La fête parce qu'elle est collective, le mawlid parce qu'il est hétérodoxe. Un premier texte ${ }^{1}$ fut alors rédigé dans lequel la fête servait de fil conducteur pour approcher la mémoire et l'imaginaire des groupes locaux ; elle permettait également de lire et de sentir autrement l'habitat.

Marc Gaborieau, qui participait à un colloque à Oran où j'avais exposé une partie de ce texte, m'avait conseillé de prendre contact avec Bernard Lepetit, alors secrétaire des Annales. Ce dernier avait particulièrement apprécié une formule à propos de l'espace que je considérais comme "du temps solidifié ». Il lira ce premier texte, qui deviendra un chapitre de ma thèse, avec de l'intérêt mais avec réserve. Cependant, sensible à ma démarche et à mon "bricolage méthodologique $»^{2}$, il m'encouragera à poursuivre et acceptera de diriger mon travail dans le cadre d'une thèse.

17 Au-delà de ce qui avait retenu son attention, Bernard Lepetit me fit un jour, à peu près en ces termes, la critique suivante: "L'intérêt n'est pas de découvrir sur le terrain ce qui fonctionne dans votre modèle pré-construit, mais précisément ce qui ne fonctionne pas. »

18 Cette remarque, fondamentale à mon sens, modifiera sensiblement mon regard et ma démarche. J'étais parti pour étudier l'espace au sens physique, j'ai fini par tenir compte également de l'espace socio-historique. Je voulais étudier les invariants, j'ai fini par questionner le changement. J'ai, enfin, pu réaliser combien le passé et le présent sont imbriqués; et combien, sous l'apparente immobilité des choses, le frémissement d'un mouvement incessant est perceptible à celui qui sait écouter. Bernard Lepetit savait écouter.

\section{NOTES}

1. «Un mawlid à Kenadsa : la fête ou le génie du lieu », document URASC, 1991, 31 p. 
2. Au lieu d'interviews classiques, j'avais opté pour l'observation participante. J'avais recueilli des récits et des légendes dont j'ai tenté d'analyser le contenu en usant de ressources multiples, explorant tous les indices, aussi bien les manuscrits et les documents écrits que les mythes, les récits oraux, les chants et les prières.

\section{AUTEUR}

\section{ABDERRAHMANE MOUSSAOUI}

EHESS, sous la direction de Bernard Lepetit : Thèse soutenue, février 1996, Logiques du sacré et modes d'organisation de l'espace dans le Sud-Ouest algérien.

Chercheur URASC, université d'Oran 